\title{
Accuracy of Intensity and Inclinometer Output of Three Activity Monitors for Identification of Sedentary Behavior and Light-Intensity Activity
}

\author{
Lucas J. Carr and Matthew T. Mahar \\ Activity Promotion Laboratory, Department of Kinesiology, East Carolina University, Greenville, NC 27858, USA \\ Correspondence should be addressed to Lucas J. Carr, carrl@ecu.edu
}

Received 31 May 2011; Revised 8 August 2011; Accepted 25 August 2011

Academic Editor: Patty Freedson

Copyright (C) 2012 L. J. Carr and M. T. Mahar. This is an open access article distributed under the Creative Commons Attribution License, which permits unrestricted use, distribution, and reproduction in any medium, provided the original work is properly cited.

Purpose. To examine the accuracy of intensity and inclinometer output of three physical activity monitors during various sedentary and light-intensity activities. Methods. Thirty-six participants wore three physical activity monitors (ActiGraph GT1M, ActiGraph GT3X+, and StepWatch) while completing sedentary (lying, sitting watching television, sitting using computer, and standing still) light (walking $1.0 \mathrm{mph}$, pedaling $7.0 \mathrm{mph}$, pedaling $15.0 \mathrm{mph}$ ) intensity activities under controlled settings. Accuracy for correctly categorizing intensity was assessed for each monitor and threshold. Accuracy of the GT3X+ inclinometer function (GT3X+Incl) for correctly identifying anatomical position was also assessed. Percentage agreement between direct observation and the monitor recorded time spent in sedentary behavior and light intensity was examined. Results. All monitors using all thresholds accurately identified over $80 \%$ of sedentary behaviors and $60 \%$ of light-intensity walking time based on intensity output. The StepWatch was the most accurate in detecting pedaling time but unable to detect pedal workload. The GT3X+Incl accurately identified anatomical position during $70 \%$ of all activities but demonstrated limitations in discriminating between activities of differing intensity. Conclusions. Our findings suggest that all three monitors accurately measure most sedentary and light-intensity activities although choice of monitors should be based on study-specific needs.

\section{Introduction}

Physical inactivity is a leading preventable cause of allcause mortality [1] and has been identified as one of the most important public health problems of the 21st century [2]. Physical inactivity has traditionally been considered the absence of physical activity at the moderate-to-vigorous intensity level which mistakenly combines the large portion of time individuals spend engaged in sedentary behaviors and light-intensity activities.

Sedentary behavior is defined as those activities that do not increase energy expenditure substantially above the resting level (i.e., 1.0-1.5 metabolic equivalent units (METs) [3] and includes activities such as sleeping (0.95 METs), lying down (1.0-1.3 METs), sitting watching television (1.3 METs), sitting at a computer doing light office work (1.5 METs), and standing still quietly (1.3 METs) [4]. Lightintensity physical activity has been described as activities that result in energy expenditure at the level of 1.6-2.9 METs such as walking slower than $2.0 \mathrm{mph}(2.0 \mathrm{METs})$, cooking food (2.0 METs), and washing dishes (1.8 METs) [4].

Recent research has begun to explore more deeply into specific health implications associated with prolonged sedentary behaviors. For instance, in a cross-sectional study of 1,958 older men and women residing in Australia, both self-reported sitting time and television viewing time were associated with increased risk for metabolic syndrome [5]. In a subsample of the same study $(n=168)$, objectively measured sedentary time (via accelerometer) was found to be independently associated with increased waist circumference and risk for metabolic disease [6], whereas more breaks in sedentary time were beneficially associated with waist circumference, body mass index (BMI), triglycerides, and 2h plasma glucose [7]. Consistently, objectively assessed sedentary time (via physical activity monitors) has also been 
associated with increased biomarkers of chronic diseases (e.g., BMI, waist circumference, C-reactive protein, fasting insulin, and homeostasis model assessment of insulin resistance) in a sample of postmenopausal women [8]. Recent evidence that suggests replacing 30 minutes/day of sedentary time with light-intensity physical activity is associated with better physical health and overall well-being [9] highlights the need to have a thorough understanding of the differences between sedentary and light-intensity activity.

While evidence accumulates implicating reduced sedentary time as a target for interventions, there is still disagreement as to best practices for measuring sedentary behaviors. Although methods for measuring physical activity have become more sophisticated, methods for measuring sedentary behaviors lag behind. Early efforts to estimate sedentary time utilized self-report measures, which are critical for assessing population trends and effectiveness of public health interventions. However, self-report measures are often limited in their accuracy due to self-report bias and often do not target all sedentary behaviors [10]. Further, many selfreport measures of sedentary time focus on specific activities such as television viewing time, limiting the interpretation of findings [10].

Objective measures, such as physical activity monitors, which have traditionally been utilized to measure physical activity of light-to-vigorous intensity, offer functionality that can also be used to measure sedentary behaviors. For example, as technology develops, new physical activity monitors are becoming available that have unique features (e.g., location, software analysis features, inclinometer, etc.) that may allow for better assessment of sedentary behavior. However, different physical activity monitors also have unique limitations and standardized procedures are still in development. For instance, there remains disagreement with regards to appropriate thresholds for calculating sedentary behavior.

Two physical activity monitors that have been widely used to date include the StepWatch physical activity monitor (Orthocare Innovations, Mountlake Terrace, Wash, USA) and ActiGraph accelerometer-based activity monitors (ActiGraph LLC, Pensacola, Fla, USA). ActiGraph has been on the forefront of accelerometry technology and has developed multiple models of physical activity monitors including previously demonstrated [11-14] single-axis GT1M and the more recent triaxial GT3X+ model which has unique functionalities, some of which have yet to be extensively tested.

Evidence of the validity of the ActiGraph accelerometerbased activity monitor to assess levels of moderate-tovigorous physical activity has been presented [11-14], but limited research has examined the validity of the ActiGraph for assessment of sedentary behavior. Matthews et al. applied a threshold of $<100$ counts/minute from ActiGraph accelerometer-based activity monitors to a large representative sample from the 2003-2004 NHANES data set to represent sedentary behavior. The study authors reported that participants spent 55\% of their time (approximately 7.7 hours/day) engaged in sedentary behaviors [15]. The GT1M was also recently demonstrated to correlate significantly, but modestly with self-reported sedentary activities (watching television (TV), computer use, reading, socializing, transport and hobbies, and a summary measure (total sedentary time)) amongst 48 older adults [16].

The tri-axis ActiGraph GT3X+ accelerometer-based activity monitor offers a unique combination of both vector magnitude calculation, allowing for measurement of physical activity intensity in three separate planes, and inclinometer functionality, allowing for the identification of anatomical position (e.g., lying down, sitting, standing). Kozey-Keadle et al. recently reported a correlation between directly observed sedentary minutes and sedentary minutes from the GT3X using the 100 counts/minute threshold of 0.62 . Their results suggested that a threshold of 150 counts/minute provided a better assessment of sedentary behavior, relative to direct observation [17]. However, to date, the validity of the GT3X+ vector magnitude and inclinometer functions for distinguishing between sedentary and light intensity activities has yet to be demonstrated.

The StepWatch physical activity monitor is worn on the ankle, as opposed to the hip placement of the ActiGraph, allowing for possible differences in its ability to measure sedentary and physically active behaviors. Evidence of the accuracy and validity of the StepWatch has previously been demonstrated during controlled laboratory settings for measuring walking behavior and estimating energy expenditure $[18,19]$, although the ability of the StepWatch to distinguish between sedentary, light- and moderate-intensity activities is also unknown.

Currently, there are few valid objective measures for assessing sedentary behaviors. It is necessary to test the accuracy of the available physical activity monitors for their ability to accurately distinguish between the spectrums of physical activity intensities. Therefore, the primary aim of the present study was to examine the accuracy of intensity output of three physical activity monitors (GT3X+, GT1M, and StepWatch) for measurement of sedentary behavior and light-intensity activities under controlled settings. A secondary aim was to examine the accuracy of the inclinometer output of the GT3X+ monitor for measurement of anatomical position. We anticipated all three monitors to be accurate for measurement of sedentary activities under controlled settings. We also anticipated differences between the monitors for accurately measuring pedaling activities of light and moderate intensity due to differing placement (hip versus ankle) of the three physical activity monitors. It is anticipated that the findings of this study will provide researchers a guide for choosing appropriate monitors for the measurement of sedentary behavior and light-intensity activities in future studies.

\section{Materials and Methods}

2.1. Subjects. A total of 36 healthy, college-aged adults (age $=23.0 \pm 3.7$ years; BMI $=23.8 \pm 3.8 \mathrm{~kg} / \mathrm{m}^{2} ; 55 \%$ female) were recruited for participation (see Table 1 ) and completed all study requirements. The BMI's of the included sample ranged from 19.7 to $39.1 \mathrm{~kg} / \mathrm{m}^{2}$ with $30.5 \%$ of the sample categorized as overweight/obese. Completion of the study 
TABLe 1: Demographic characteristics of sample $(n=36)$.

\begin{tabular}{lcc}
\hline & Mean & St. dev. \\
\hline Age (years) & 23.0 & 3.7 \\
Height $(\mathrm{cm})$ & 171.2 & 9.5 \\
Weight $(\mathrm{kg})$ & 70.6 & 15.8 \\
BMI $\left(\mathrm{kg} / \mathrm{m}^{2}\right)$ & 23.8 & 3.8 \\
Female $(\%)$ & $55.0 \%$ & \\
Racial group $(\%)$ & & \\
$\quad$ American Indian/Alaskan Native & $0.0 \%$ & \\
$\quad$ Asian & $2.8 \%$ & \\
$\quad$ Native Hawaiian or other Pacific Islander & $0.0 \%$ & \\
$\quad$ Black or African American & $19.4 \%$ & \\
$\quad$ White & $69.4 \%$ & \\
$\quad$ Do not know/refuse & $8.3 \%$ & \\
Ethnicity $(\%)$ & & \\
$\quad$ Hispanic & $2.0 \%$ & \\
$\quad$ Non-Hispanic & $68.0 \%$ & \\
$\quad$ Do not know/refuse & $30.0 \%$ & \\
\hline
\end{tabular}

was defined as wearing three physical activity monitors during six consecutive activities under controlled settings. Participants were devoid of any ambulatory limitations that would prevent them from engaging in the activities for eight consecutive minutes. Participants were not compensated for participation in this study. Experimental protocols were approved by the University and Medical Center Institutional Review Board at the authors' institution and voluntary informed consent was obtained from each participant.

2.2. Experimental Design. All testing assessments were conducted during the months of March and April 2011. Following the informed consent, participants were asked to attend one 60-minute testing session. During this session, participants were asked to complete a series of six consecutive activities that varied in both movement (i.e., no movement, walking, and pedaling) and anatomical position (i.e., lying, sitting, and standing). Activities were identified as either sedentary or light intensity according to the most recent Physical Activity Compendium [4]: (1) lying still with legs straight and flat on back in a horizontal position (estimated 0.9 METS); (2) sitting relaxed but upright in a chair while watching television with feet on floor (estimated 1.0 METS); (3) sitting relaxed but upright in a chair with feet on the floor while using a computer (estimated 1.5 METS); (4) standing upright, while completely still (estimated 1.2 METS); (5) walking very slowly at $50 \mathrm{steps} / \mathrm{min}$ or roughly $1.0 \mathrm{mph}$ (estimated 2.0 METS); (6) pedaling on a magnetic resistance pedal exercise machine (MagneTrainer; 3D Innovations, LLC, Greeley, CO) at either a slow pace $(7.0 \mathrm{mph} ; n=19)$ or a self-selected pace (recorded mean $=15.0 \mathrm{mph} ; n=17)$. The activities used in this study were chosen to represent a range of activities (sedentary lying down to low-intensity cycling) that are often performed in free-living environments (lying down, TV viewing, computer use, and walking), but differed distinctly in both movements (no movement versus walking versus cycling) and anatomical positions (lying down, sitting, and standing).

Walking was guided by a metronome set at $50 \mathrm{steps} / \mathrm{min}$ and participants were observed walking at this pace. All participants were instructed to pedal at a constant speed guided by a speedometer on the MagneTrainer. Participants who pedaled at self-selected speeds were instructed to identify a "comfortable" pedal pace within the first two minutes and remain at that pace for the remainder of the activity. The resistance level of the pedal machines was consistent for both pedaling cadences. The intensity of the two pedal cadences was confirmed as light intensity based on both heart rate and rating of perceived exertion (RPE) data with a pilot sample $(N=10)$ of participants of a similar age (23.5 years) to the study sample.

Each activity lasted eight minutes in duration with twominute breaks in between each activity. All participants were instructed to complete each task in the same anatomical position. To address protocol fidelity (i.e., to ensure 100\% time participants spent in each activity was true to the activity), activities were observed directly and the first and last minute of each activity were removed from analyses leaving six minutes for each activity.

To allow analysis of the same activity by multiple assessments, participants completed the six activities while wearing three different physical activity monitors: (1) the ActiGraph GT3X+; (2) the ActiGraph GT1M; (3) the StepWatch. These three monitors were purposefully tested to compare multiple monitors of differing placement (hip versus ankle) and functionality (e.g., step rate, accelerometry, and inclinometer) with the hope of adding to the knowledge based on specific monitors that are best suited for measuring specific sedentary and light-intensity activities. For example, many physical activity intervention studies encourage walking [20] while others encourage cycling [21]. It is important that researchers choose objective monitors that most accurately assess the primary activity being promoted or measured. Participants wore the two ActiGraph monitors on a belt over their left or right hips and the StepWatch on either their left or right ankle. The locations of all three monitors were counterbalanced to result in an equal number of participants wearing each monitor on the left and right sides of their body.

The StepWatch was initialized based on the participant's self-reported height and steps per minute were recorded. The GT1M monitor was initialized at 1-second epochs and reintegrated to 60 -second epochs to calculate counts per minute to remain consistent with the StepWatch. The GT3X+ monitor was initialized at a frequency of $30 \mathrm{~Hz}$ and counts/min was then calculated to remain consistent with the other two monitors. The low-frequency extension (LFE) was not selected when initializing the GT3X+. Minutes spent in each of the six different activities were then separated by each monitor to allow for specific monitor/activity analyses. Summary files were then created to calculate the total number of minutes out of six minutes correctly recorded as either sedentary behavior (lying down, sitting watching television, sitting and typing on computer, and standing still) or light intensity (walking slowly at $1.0 \mathrm{mph}$, pedaling at 7.0 or $15.0 \mathrm{mph}$ ). 
The thresholds used for each monitor are provided in Table 2. The sedentary threshold used for the StepWatch (zero) was based on the recommendation provided by the product manufacturer (StepWatch). The thresholds for light(1-45 steps/min) and moderate- (46+ steps/min) intensity physical activity used for the StepWatch were based on previous work which demonstrated moderate-intensity stride rate to range from 90-113 steps/minute depending on height and stride length $[22,23]$.

Based on results from Kozey-Keadle et al., two different thresholds (i.e., $<100$ counts/minute and $<150$ counts/min) were used for calculating sedentary time from the GT1M (GT1M100, GT1M150) and GT3X+ (GT3X+100, GT3X+150) monitors [17]. Counts per minute were collected from the single axis for the GT1M and from the vector magnitude for the GT3X+ monitors. No thresholds for sedentary have been established for the GT3X+. Previously established thresholds developed on the GT1M [15, 17] were used to categorize activities as either light or moderate intensity for both the GT1M and GT3X+ monitors.

The GT3X+ inclinometer function $(\mathrm{GT} 3 \mathrm{X}+\mathrm{Incl})$ was tested for its ability to accurately identify anatomical positions (i.e., lying down, sitting, and standing) during the six activities, which is not a direct measure of the intensity of the activity. Manufacturer recommendations were followed to categorize each minute of activity into an anatomical position (i.e., 1 for standing, 2 for lying down, 3 for sitting, and 0 for nonwear).

2.3. Statistical Analysis. Validity of the activity monitors/thresholds was examined by comparing the monitorrecorded time (total minutes out of six minutes) coded as sedentary behavior, light intensity, or moderate intensity with the criterion of direct observation. For the GT3X+Incl function, validity was examined by comparing the monitor recorded time (total minutes out of six minutes) coded as lying down, sitting, standing and/or non-wear with the criterion of direct observation. Specifically, percentage of agreement was defined as the percent agreement between the criterion measure of observed intensity or anatomical position minutes and the monitor-recorded intensity or anatomical position minutes. The $95 \%$ confidence interval was calculated for the mean minutes out of 6 minutes correctly coded for both intensity and anatomical position. Significance was determined based on whether the 95\% confidence interval spanned the criterion of 6 minutes correct. Root mean square error for minutes of sedentary behaviors (lying down, sitting watching television, sitting using computer, and standing) and light-intensity activities (walking $1.0 \mathrm{mph}$, pedaling $7.0 \mathrm{mph}$, pedaling $15.0 \mathrm{mph}$ ) coded correctly out of 6 minutes was calculated for both intensity and inclinometer outputs. While statistical analyses were performed on minutes, data are reported as percent time coded in each intensity/anatomical position.

\section{Results}

The sample included healthy, college-aged adults of normalto obese-weight status (BMI range $=19.7-39.1 \mathrm{~kg} / \mathrm{m}^{2}$ ) and a roughly even gender distribution (see Table 1). Participants were primarily White $(69.4 \%)$ or Black/African American (19.4\%) and the majority were non-Hispanic (68.0\%).

When examining the minutes correctly identified as sedentary, all three physical activity monitors accurately identified over $80 \%$ of all sedentary activities (lying down, sitting watching television, sitting using computer, and standing still) as sedentary using multiple thresholds for sedentary behavior (see Figure 1, Table 3 ).

When examining minutes correctly identified as light intensity, all three monitors/thresholds accurately identified at least $70 \%$ of all walking minutes as light intensity with the exception of the GT1M150 (60.7\%). The StepWatch was the most accurate identifying $88.8 \%$ of slow walking time as light intensity, whereas the GT1M150 was the least accurate $(60.7 \%$ agreement). For the pedaling activities, the StepWatch was the only monitor to accurately record more than half of all pedaling time at $7.0 \mathrm{mph}(54.4 \%)$ and $15.0 \mathrm{mph}(100 \%)$ as "nonsedentary". However, $45.6 \%$ of pedaling time at $7.0 \mathrm{mph}$ was incorrectly coded as sedentary and $60.8 \%$ of pedaling time at $15.0 \mathrm{mph}$ was incorrectly coded as moderate intensity with the StepWatch.

The inclinometer function of the GT3X+ correctly identified anatomical position between 60.6 and $66.7 \%$ of the time during the four sedentary activities (see Table 4 ). The GT3X+Incl also accurately identified $71.8 \%$ of light-intensity walking time as standing, $85.1 \%$ of pedaling at $7.0 \mathrm{mph}$ as sitting, and $74.5 \%$ of pedaling at $15.0 \mathrm{mph}$ as sitting. Across all sedentary and light-intensity activities, the GT3X+Incl coded less than $14.3 \%$ of all wear time as zero or "non-wear".

Root mean square error for minutes correctly coded as sedentary behavior and light-intensity activity are presented in Table 5. Root mean square error was lowest for the GT1M150 (0.07) and highest for the GT3X+100 monitor (0.86) during sedentary behaviors while root mean square error was lowest for the GT1M150 (2.10) and highest for the StepWatch monitor (3.33) during light-intensity activities. The root mean square error was consistently larger for light-intensity activities as compared to sedentary behaviors across all monitors for intensity outputs. Root mean square error for correctly identifying minutes in anatomical position was larger during sedentary behaviors than light-intensity activities for the GT3X+Inclinometer function (see Table 5).

\section{Discussion}

The findings from this study support the StepWatch, GT1M, and GT3X+ physical activity monitors as accurate measures of time spent sedentary based on intensity output during such activities as lying down, sitting watching television, sitting using computers, and standing still under controlled settings. When comparing average accuracy for correctly coding the four sedentary behaviors across all monitors/thresholds, the GT1M monitor was the most accurate when using the $<150$ counts/min threshold (average $99.2 \%$ ) followed by the GT1M at the $<100$ threshold (average 98.4\%), the StepWatch (average 96.2\%), GT3X+ vector magnitude at the $<150$ threshold (average 90.1\%), and the GT3X+ 
TABLE 2: Thresholds for intensity and anatomical positions for each physical activity monitor.

\begin{tabular}{|c|c|c|c|}
\hline & Sedentary & Light intensity & Moderate intensity \\
\hline StepWatch & 0 steps/min & $1-45$ steps/min & $46+$ steps $/ \mathrm{min}$ \\
\hline GT1M100 & 0-99 counts/min & $100-2220$ counts/min & $2221+$ counts $/ \mathrm{min}$ \\
\hline GT1M150 & $0-149$ counts/min & $150-2220$ counts $/ \mathrm{min}$ & $2221+$ counts $/ \mathrm{min}$ \\
\hline GT3X+100 & 0-99 counts/min & $100-2689$ counts/min & $2690+$ counts $/ \mathrm{min}$ \\
\hline GT3X+150 & $0-149$ counts $/ \mathrm{min}$ & $150-2689$ counts $/ \mathrm{min}$ & $2690+$ counts $/ \mathrm{min}$ \\
\hline \multirow[t]{4}{*}{ GT3X+Incl } & Coded $1=$ standing still & Coded $1=$ walking & \\
\hline & Coded $2=$ lying down & Coded $3=$ sitting pedaling & \\
\hline & Coded $3=$ sitting TV/computer & Coded $0=$ Non-wear & \\
\hline & Coded $0=$ Non-wear & & \\
\hline
\end{tabular}

vector magnitude at the $<100$ threshold $(87.0 \%)$. While statistical significance was often detected when comparing the monitor-recorded times against the criterion of $100 \%$ accuracy, such findings should be interpreted with caution. Small differences are often statistically significant due to large sample size and small sample variation. For example, monitors that were highly accurate at identifying a particular intensity had very little variability on the identified activity codes. The agreement between the activity recorded by the monitors and the directly observed criterion measures provides a guide for researchers trying to choose the best measure of sedentary time. Overall, these findings support the use of all three physical activity monitors for assessing time spent sedentary with little difference between thresholds of 100 and 150 counts/min.

All three monitors also appear to be fairly accurate in identifying slow walking at $1.0 \mathrm{mph}$ as light-intensity activity. With the exception of the GT1M when using the 150 counts/min threshold, all monitors/thresholds accurately identified over $70 \%$ of all slow walking time as light intensity. It appears that the GT1M was less accurate when using the $<150$ counts/min threshold to discriminate between sedentary behaviors and light-intensity activities. This example, however, illustrates an important point in that measurement of physical activity intensity depends on the chosen threshold and researchers should be aware that there is a trade-off with using such thresholds. For example, when comparing the two thresholds used for discriminating between sedentary and light-intensity activity $(<100$ and $<150$ counts/min) for the GT1M and the GT3X+, it appears that expanding the definition of sedentary to all activities under 150 counts/minute increases the percent agreement slightly for sedentary activities for both monitors which is consistent with previous studies [17]. Specifically, the GT1M and GT3X+ monitors accurately identified an average of $99.2 \%$ and $90.1 \%$ of all sedentary minutes when using the 150 counts/min thresholds, respectively.

Percent agreement for identifying walking as lightintensity dropped $10 \%$ for the GT1M when using the 150 counts/min threshold compared to the 100 counts/min threshold, while the percent agreement for the GT3X+ was nearly identical for both thresholds. Therefore, the effect of changing the activity count threshold may be that the

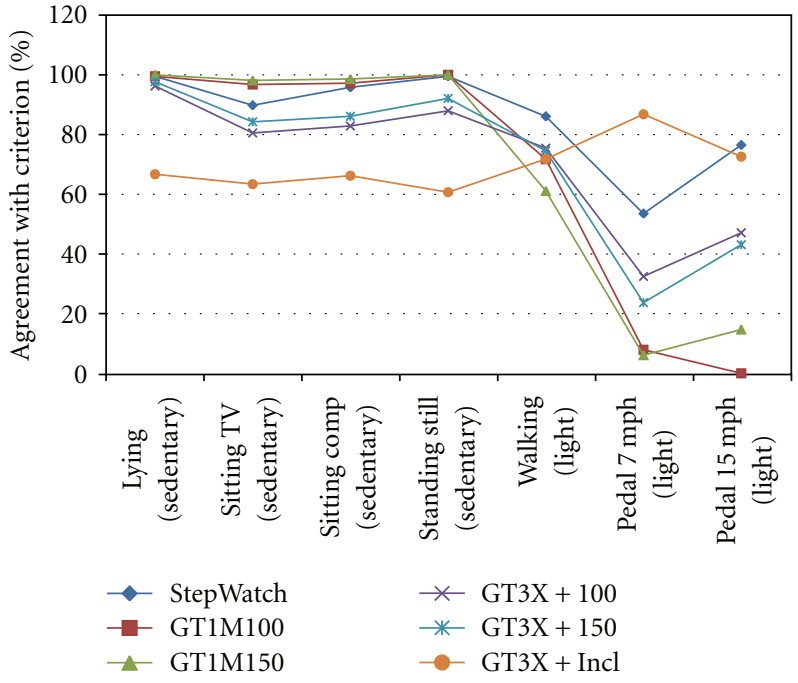

Figure 1: Concurrent time interval of mean percent agreement for intensity (StepWatch, GT1M100, GT1M150, GT3X+100 and GT3X+150) and anatomical position (GT3X+Incl) across all activities.

accuracy of time spent in one intensity category (e.g., sedentary) improves, while the accuracy of identifying time spent in the adjacent category (e.g., light-intensity) is sacrificed. Additionally, it is possible that the accuracy of specific thresholds is dependent upon additional factors, such as levels of adiposity, and these potential influences should be explored.

Additionally, specific to the Actigraph monitors, measurement of sedentary time is also dependent upon specific features such as the low-frequency extension (LFE), which is intended to broaden the low-frequency range of the digital band-pass filter allowing for more sensitive detection of movement. Although the LFE was not enabled in the present study, the GT3X+ appears to be more sensitive to movement than the single-axis GT1M. This suggests that different thresholds for sedentary behavior may be appropriate for the two monitors. Based on the present findings it appears that 100 counts/min is more appropriate for the GT1M, whereas 150 counts/minute provides slightly more accurate identification of sedentary behavior and lightintensity activity than the 100 counts/min threshold for the 
Table 3: Percent time coded as sedentary behavior, light intensity, moderate intensity, and mean coded correctly ( $\pm 95 \%$ CI) for each monitor/threshold and activity.

\begin{tabular}{|c|c|c|c|c|c|c|}
\hline Stepwatch & Correct code & $\begin{array}{l}\text { Mean coded } \\
\text { correct }(\%)\end{array}$ & $\begin{array}{c}95 \% \text { confidence } \\
\text { interval }\end{array}$ & $\begin{array}{c}\% \text { coded } \\
\text { sedentary }\end{array}$ & $\%$ coded light & $\begin{array}{l}\% \text { coded } \\
\text { moderate }\end{array}$ \\
\hline Lying down & Sedentary & 99.5 & $98.6-100.5$ & 99.5 & 0.5 & - \\
\hline Sitting TV & Sedentary & 89.8 & $82.9-96.7$ & 89.8 & 10.2 & - \\
\hline Sitting computer & Sedentary & 95.8 & $92.7-99.0$ & 95.8 & 4.2 & - \\
\hline Standing & Sedentary & 99.5 & $98.6-100.5$ & 99.5 & 0.5 & - \\
\hline Walking (1.0 mph) & Light & 86.1 & $76.8-95.4$ & 3.8 & 88.8 & 7.4 \\
\hline Pedaling $7.0 \mathrm{mph}$ & Light & 54.4 & $35.7-73.1$ & 45.6 & 54.4 & 0.0 \\
\hline Pedaling $15.0 \mathrm{mph}$ & Light & 23.5 & $8.8-38.3$ & 0.0 & 39.2 & 60.8 \\
\hline \multicolumn{7}{|l|}{ GT1M100 } \\
\hline Lying down & Sedentary & 99.5 & $98.6-100.5$ & 99.5 & 0.5 & - \\
\hline Sitting TV & Sedentary & 96.8 & $93.8-99.7$ & 96.8 & 3.2 & - \\
\hline Sitting computer & Sedentary & 97.2 & $94.7-99.7$ & 97.2 & 2.8 & - \\
\hline Standing & Sedentary & 100.0 & $100.0-100.0$ & 100.0 & 0.0 & - \\
\hline Walking (1.0 mph) & Light & 71.8 & $59.4-84.1$ & 28.2 & 71.8 & 0.0 \\
\hline Pedaling $7.0 \mathrm{mph}$ & Light & 8.1 & $-2.5-18.6$ & 92.1 & 7.9 & 0.0 \\
\hline Pedaling $15.0 \mathrm{mph}$ & Light & 15.5 & $0.8-30.1$ & 88.5 & 11.5 & 0.0 \\
\hline \multicolumn{7}{|l|}{ GT1M150 } \\
\hline Lying down & Sedentary & 100.0 & $100.0-100.0$ & 100.0 & 0.0 & - \\
\hline Sitting TV & Sedentary & 98.2 & $96.4-100.0$ & 98.2 & 1.8 & - \\
\hline Sitting computer & Sedentary & 98.6 & $97.0-100.2$ & 98.6 & 1.4 & - \\
\hline Standing & Sedentary & 100.0 & $100.0-100.0$ & 100.0 & 0.0 & - \\
\hline Walking (1.0 mph) & Light & 61.1 & $47.7-74.5$ & 39.3 & 60.7 & 0.0 \\
\hline Pedaling $7.0 \mathrm{mph}$ & Light & 6.6 & $-4.0-17.1$ & 93.9 & 6.1 & 0.0 \\
\hline Pedaling $15.0 \mathrm{mph}$ & Light & 14.7 & $0.0-29.4$ & 85.3 & 14.7 & 0.0 \\
\hline \multicolumn{7}{|l|}{ GT3X+100 } \\
\hline Lying down & Sedentary & 96.3 & $93.0-99.6$ & 96.3 & 3.7 & - \\
\hline Sitting TV & Sedentary & 80.6 & $69.7-91.4$ & 80.6 & 19.4 & - \\
\hline Sitting computer & Sedentary & 82.9 & $74.7-91.0$ & 82.9 & 17.1 & - \\
\hline Standing & Sedentary & 88.0 & $80.6-95.3$ & 88.0 & 12.0 & - \\
\hline Walking (1.0 mph) & Light & 75.5 & $64.3-86.6$ & 18.0 & 75.5 & 6.5 \\
\hline Pedaling $7.0 \mathrm{mph}$ & Light & 33.2 & $18.5-47.9$ & 67.5 & 32.5 & 0.0 \\
\hline Pedaling $15.0 \mathrm{mph}$ & Light & 47.1 & $26.5-67.6$ & 52.0 & 47.1 & 0.9 \\
\hline \multicolumn{7}{|l|}{ GT3X+150 } \\
\hline Lying down & Sedentary & 97.7 & $95.3-100.1$ & 97.7 & 2.3 & - \\
\hline Sitting TV & Sedentary & 84.3 & $74.4-94.2$ & 84.3 & 15.7 & - \\
\hline Sitting computer & Sedentary & 86.1 & $79.5-92.8$ & 86.1 & 13.9 & - \\
\hline Standing & Sedentary & 92.1 & $85.7-98.5$ & 92.1 & 7.9 & - \\
\hline Walking (1.0 mph) & Light & 74.5 & $62.5-86.6$ & 19.0 & 74.5 & 6.5 \\
\hline Pedaling $7.0 \mathrm{mph}$ & Light & 23.7 & $8.9-38.4$ & 76.3 & 23.7 & 0.0 \\
\hline Pedaling $15.0 \mathrm{mph}$ & Light & 43.1 & $21.7-64.6$ & 55.9 & 43.1 & 1.0 \\
\hline
\end{tabular}

GT3X+. These findings are consistent with recent findings by Kozey-Keadle et al [17].

When examining pedal activity, consistent with our hypothesis, the ankle-worn StepWatch was the only monitor to identify more than half of pedaling time as nonsedentary. This makes intuitive sense as both ActiGraph monitors are worn on the hip and past studies have confirmed waistmounted pedometers to be less sensitive to lower-extremity pedaling activity than ankle-worn monitors such as the StepWatch [24]. Still, while the StepWatch was able to pick up more pedal activity than the Actigraph monitors, the StepWatch can only code intensity based on pedal rate rather than workload. This is illustrated in the present study by the large discrepancy in intensity identification between the two pedal rates of 7.0 and $15.0 \mathrm{mph}$ which were performed at the same low resistance. These findings indicate 
TABle 4: Percent time anatomical position was coded correctly ( $\pm 95 \%$ confidence interval) and incorrectly (as lying, sitting, standing, or non-wear) for the GT3X+ Inclinometer for each activity.

\begin{tabular}{|c|c|c|c|c|c|c|c|}
\hline GT3X+Incl & Correct code & $\begin{array}{c}\text { Coded } \\
\text { correct }(\%)\end{array}$ & $\begin{array}{l}95 \% \text { conf. } \\
\text { interval }\end{array}$ & $\begin{array}{c}\text { Position } \\
\text { coded lying } \\
(\%)\end{array}$ & $\begin{array}{c}\text { Position } \\
\text { coded sitting } \\
(\%)\end{array}$ & $\begin{array}{c}\text { Position } \\
\text { coded } \\
\text { standing (\%) } \\
\end{array}$ & $\begin{array}{l}\text { Position coded } \\
\text { "non-wear" (\%) }\end{array}$ \\
\hline Lying down & 2 = lying & 66.7 & $52.2-81.2$ & - & 13.9 & 5.1 & 14.3 \\
\hline Sitting TV & $3=$ sitting & 63.4 & $50.5-76.4$ & 0.0 & - & 30.1 & 6.5 \\
\hline Sitting computer & $3=$ sitting & 66.2 & $53.3-79.1$ & 0.9 & - & 23.6 & 9.3 \\
\hline Standing & $1=$ standing & 60.6 & $46.3-75.1$ & 0.0 & 37.5 & - & 1.9 \\
\hline Walking $1.0 \mathrm{mph}$ & $1=$ standing & 71.8 & $59.7-83.8$ & 0.0 & 28.2 & - & 0.0 \\
\hline Pedaling $7.0 \mathrm{mph}$ & $3=$ sitting & 85.1 & $69.7-100.4$ & 3.5 & - & 11.4 & 0.0 \\
\hline Pedaling $15.0 \mathrm{mph}$ & $3=$ sitting & 74.5 & $54.4-94.6$ & 0.0 & - & 19.6 & 5.9 \\
\hline
\end{tabular}

TABle 5: Root mean square error for minutes of sedentary behaviors (lying down, sitting watching television, sitting using computer, standing) and light intensity activities (walking $1.0 \mathrm{mph}$, pedaling $7.0 \mathrm{mph}$, pedaling $15.0 \mathrm{mph}$ ) coded correctly out of 6 possible minutes.

\begin{tabular}{lcccccc}
\hline & StepWatch & GT1M100 & GT1M150 & GT3X+100 & GT3X+150 & GT3X+Incl \\
\hline Sedentary behaviors & 0.33 & 0.13 & 0.07 & 0.86 & 0.68 & 2.15 \\
Light Intensity activities & 3.33 & 2.49 & 2.10 & 2.84 & 2.81 & 0.98 \\
\hline
\end{tabular}

that although the ankle-worn StepWatch may be the most accurate of the three monitors at measuring low-intensity pedaling activity, a large portion of time spent pedaling at $15.0 \mathrm{mph}$ was inaccurately coded as moderate intensity while a large portion of pedaling time at $7.0 \mathrm{mph}$ was inaccurately coded as sedentary behavior. Researchers are encouraged to supplement StepWatch data with another measure of intensity such as heart rate or rating of perceived exertion (RPE) to better distinguish between sedentary behaviors and light-intensity behaviors.

When assessing the accuracy of the GT3X+Incl for measuring time spent in each of the three anatomical positions (e.g., lying down, sitting, and standing), our findings indicate that the inclinometer accurately identified anatomical position more than $60 \%$ of the time during sedentary behaviors. Our findings suggest the inclinometer function to be more accurate at identifying the correct position during sedentary activities of sitting and standing and less accurate at measuring time spent lying down than previously reported [25]. Further, the inclinometer function was more accurate at identifying the correct anatomical position during lightintensity activities (71.8-85.1\% percent agreement) than during sedentary activities. It appears that the inclinometer is most accurate during times of more movement which seems to result in less time coded as "non-wear".

While the inclinometer offers unique functionality in that it allows for differentiating between time spent in various anatomical positions, researchers should be cautious when using this feature. For example, when using the intensity output of the GT3X+ during pedaling time, more than half of all time was inaccurately identified as sedentary while the inclinometer accurately identified more than $74 \%$ of this same time as "sitting". Under controlled settings, the researcher could easily identify the times spent pedaling and code this time as nonsedentary but when such monitors are used in the field, without an additional measure of intensity such as heart rate monitors, the researcher would have no way of determining whether this time was sedentary or nonsedentary. This is further illustrated in the present study when comparing time spent standing still versus time spent standing and walking slowly. The GT3X+ intensity output accurately identified these times as sedentary and light intensity, respectively, whereas the inclinometer function could not distinguish between the two different activities. In summary, it seems that if researchers are interested in using the inclinometer output to identify anatomical position during sedentary behavior, our data indicate the inclinometer function only identifies roughly two-thirds of time in the correct anatomical position. Therefore, further work is warranted to identify an appropriate way to use both the intensity and the inclinometer output of the GT3X+ in combination.

The present study has several limitations. First, the findings presented are limited to the sample of relatively lean college-aged adults. The findings are also limited to the types of sedentary and light-intensity activities completed and the positions participants were in while completing each activity. For example, while sitting using the computer, participants were instructed to sit upright in a chair with their feet flat on the floor. While this is a likely position an individual would sit in while using a computer, it is possible that different results would be found if a person were sitting in a different position. This study is also limited by the vector magnitude thresholds used to estimate sedentary time for the GT3X+ monitor. Currently, no vector magnitude threshold for sedentary behavior has been established, but based on our findings, both 100 and 150 counts/min seem to provide similar results for the examined activities. 


\section{Conclusions}

Overall, all three physical activity monitors tested in this study appear to accurately identify time spent in sedentary behaviors and engaged in slow walking under controlled settings when using the intensity output. However, discrepancies appear between the monitors when measuring lowerextremity pedaling time with the ankle-worn StepWatch being the most accurate but still limited by an inability to assess pedal resistance. The GT3X+Incl seems to accurately identify anatomical position during $70 \%$ of all sedentary and light-intensity activities although this feature is also limited in its ability to distinguish between activities conducted in similar anatomical position but of differing intensity. It is recommended that researchers interested in measuring sedentary and light-intensity time use monitors with specific features that best suit the targeted activities.

\section{Acknowledgments}

The authors would like to thank Caryln Diaz, Mallory Peavler, Grace Anne Edwards, Nicholas Boerio, Mallory Clark, and Hadley Peacock, students in the Department of Kinesiology at East Carolina University, for their assistance in the completion of this study.

\section{References}

[1] A. H. Mokdad, J. S. Marks, D. F. Stroup, and J. L. Gerberding, "Actual causes of death in the United States, 2000," Journal of the American Medical Association, vol. 291, no. 10, pp. 12381245, 2004.

[2] S. N. Blair, "Physical inactivity: the biggest public health problem of the 21st century," British Journal of Sports Medicine, vol. 43, no. 1, pp. 1-2, 2009.

[3] R. R. Pate, J. R. O’Neill, and F. Lobelo, “The evolving definition of 'sedentary', Exercise and Sport Sciences Reviews, vol. 36, no. 4, pp. 173-178, 2008.

[4] B. E. Ainsworth, W. L. Haskell, S. D. Herrmann et al., "2011 compendium of physical activities: a second update of codes and MET values," Medicine and Science in Sports and Exercise, vol. 43, no. 8, pp. 1575-1581, 2011.

[5] P. A. Gardiner, G. N. Healy, E. G. Eakin et al., "Associations between television viewing time and overall sitting time with the metabolic syndrome in older men and women: the Australian diabetes obesity and lifestyle study," Journal of the American Geriatrics Society, vol. 59, no. 5, pp. 788-796, 2011.

[6] G. N. Healy, K. Wijndaele, D. W. Dunstan et al., "Objectively measured sedentary time, physical activity, and metabolic risk: The Australian Diabetes, Obesity and Lifestyle Study (AusDiab)," Diabetes Care, vol. 31, no. 2, pp. 369-371, 2008.

[7] G. N. Healy, D. W. Dunstan, J. Salmon et al., "Breaks in sedentary time: beneficial associations with metabolic risk," Diabetes Care, vol. 31, no. 4, pp. 661-666, 2008.

[8] B. M. Lynch, C. M. Friedenreich, E. A. H. Winkler et al., "Associations of objectively assessed physical activity and sedentary time with biomarkers of breast cancer risk in postmenopausal women: findings from NHANES (20032006)," Breast Cancer Research and Treatment, vol. 130, no. 1, pp. 183-194, 2011.
[9] M. P. Buman, E. B. Hekler, W. L. Haskell et al., "Objective light-intensity physical activity associations with rated health in older adults," American Journal of Epidemiology, vol. 172, no. 10, pp. 1155-1165, 2010.

[10] B. K. Clark, T. Sugiyama, G. N. Healy, J. Salmon, D. W. Dunstan, and N. Owen, "Validity and reliability of measures of television viewing time and other non-occupational sedentary behaviour of adults: a review," Obesity Reviews, vol. 10, no. 1, pp. 7-16, 2009.

[11] G. Plasqui and K. R. Westerterp, "Physical activity assessment with accelerometers: an evaluation against doubly labeled water," Obesity, vol. 15, no. 10, pp. 2371-2379, 2007.

[12] E. L. Melanson Jr. and P. S. Freedson, "Validity of the Computer Science and Applications, Inc. (CSA) activity monitor," Medicine and Science in Sports and Exercise, vol. 27, no. 6, pp. 934-940, 1995.

[13] P. S. Freedson, E. Melanson, and J. Sirard, "Calibration of the Computer Science and Applications, Inc. accelerometer," Medicine and Science in Sports and Exercise, vol. 30, no. 5, pp. 777-781, 1998.

[14] K. Zhang, P. Werner, M. Sun, F. X. Pi-Sunyer, and C. N. Boozer, "Measurement of human daily physical activity," Obesity Research, vol. 11, no. 1, pp. 33-40, 2003.

[15] C. E. Matthews, K. Y. Chen, P. S. Freedson et al., "Amount of time spent in sedentary behaviors in the United States, 20032004," American Journal of Epidemiology, vol. 167, no. 7, pp. 875-881, 2008.

[16] P. A. Gardiner, B. K. Clark, G. N. Healy, E. G. Eakin, E. A. Winkler, and N. Owen, "Measuring older adults' sedentary time: reliability, validity and responsiveness," Medicine \& Science in Sports \& Exercise, vol. 43, no. 11, pp. 2127-2133, 2011.

[17] S. Kozey-Keadle, A. Libertine, K. Lyden, J. Staudenmayer, and P. S. Freedson, "Validation of wearable monitors for assessing sedentary behavior," Medicine and Science in Sports and Exercise, vol. 43, no. 8, pp. 1561-1567, 2011.

[18] M. G. Bowden and A. L. Behrman, "Step Activity Monitor: accuracy and test-retest reliability in persons with incomplete spinal cord injury," Journal of Rehabilitation Research and Development, vol. 44, no. 3, pp. 355-362, 2007.

[19] R. C. Foster, L. M. Lanningham-Foster, C. Manohar et al., "Precision and accuracy of an ankle-worn accelerometerbased pedometer in step counting and energy expenditure," Preventive Medicine, vol. 41, no. 3-4, pp. 778-783, 2005.

[20] D. Ogilvie, C. E. Foster, H. Rothnie et al., "Interventions to promote walking: systematic review," British Medical Journal, vol. 334, no. 7605, pp. 1204-1207, 2007.

[21] L. Yang, S. Sahlqvist, A. McMinn, S. J. Griffin, and D. Ogilvie, "Interventions to promote cycling: systematic review," British Medical Journal, vol. 341, article c5293, 2010.

[22] D. A. Rowe, G. J. Welk, D. P. Heil et al., "Stride rate recommendations for moderate intensity walking," Medicine \& Science in Sports \& Exercise, vol. 43, no. 2, pp. 312-318, 2010.

[23] S. J. Marshall, S. S. Levy, C. E. Tudor-Locke et al., "Translating physical activity recommendations into a pedometer-based step goal. 3000 steps in 30 minutes," American Journal of Preventive Medicine, vol. 36, no. 5, pp. 410-415, 2009.

[24] M. Karabulut, S. E. Crouter, and D. R. Bassett Jr., "Comparison of two waist-mounted and two ankle-mounted electronic pedometers," European Journal of Applied Physiology, vol. 95, no. 4, pp. 335-343, 2005. 
[25] G. C. McMahon, R. J. Brychta, and K. Y. Chen, "Validation of the actigraph (GT3X) inclinometer function," in Medicine and Science in Sports and Exercise, American College of Sports Medicine, Baltimore, Md, USA, 2010. 


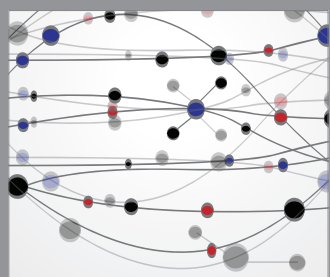

The Scientific World Journal
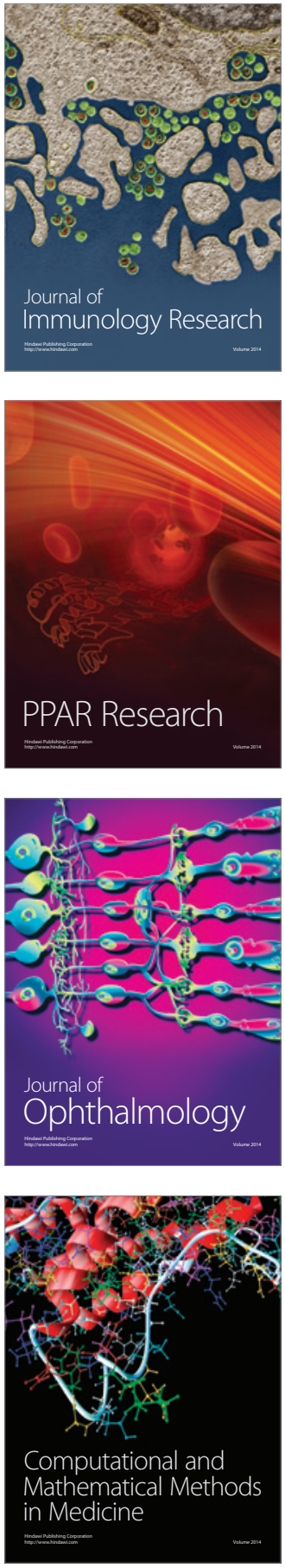

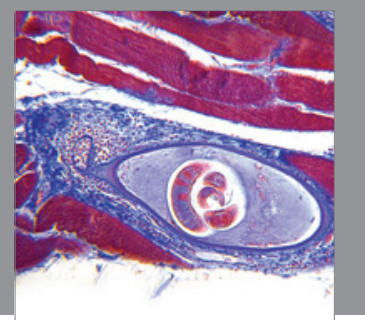

Gastroenterology

Research and Practice
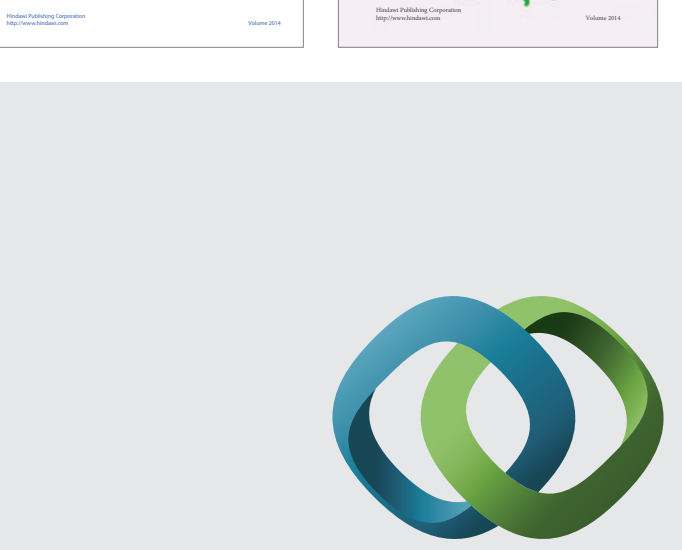

\section{Hindawi}

Submit your manuscripts at

http://www.hindawi.com
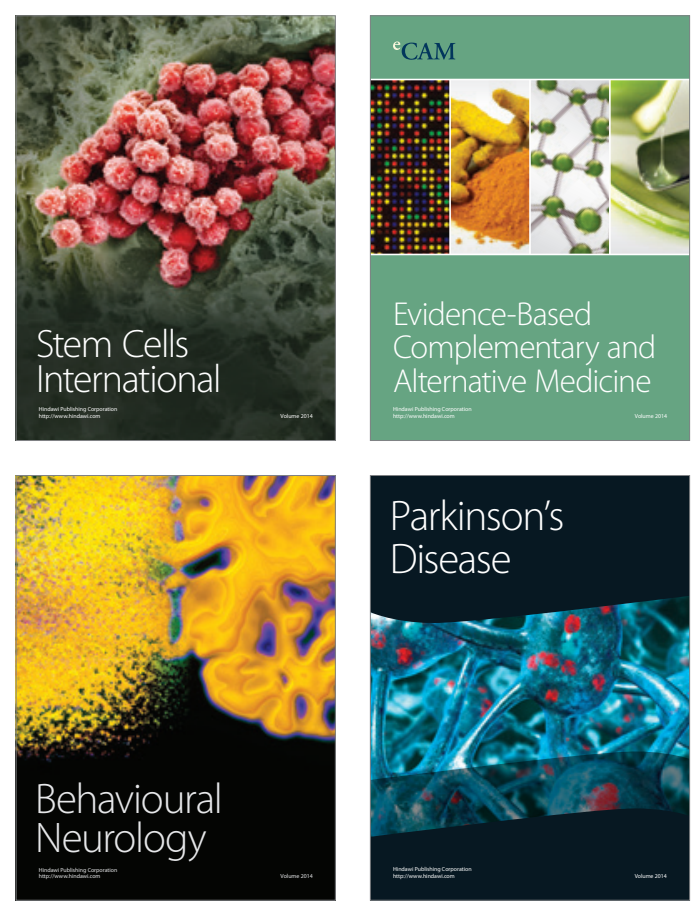

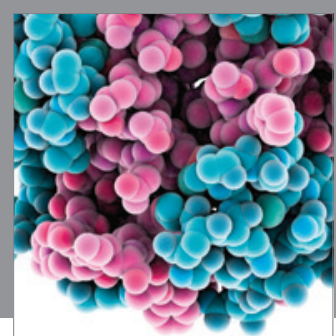

Journal of
Diabetes Research

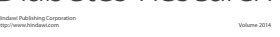

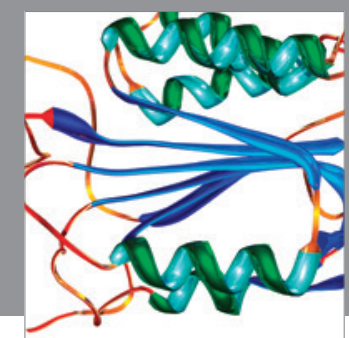

Disease Markers
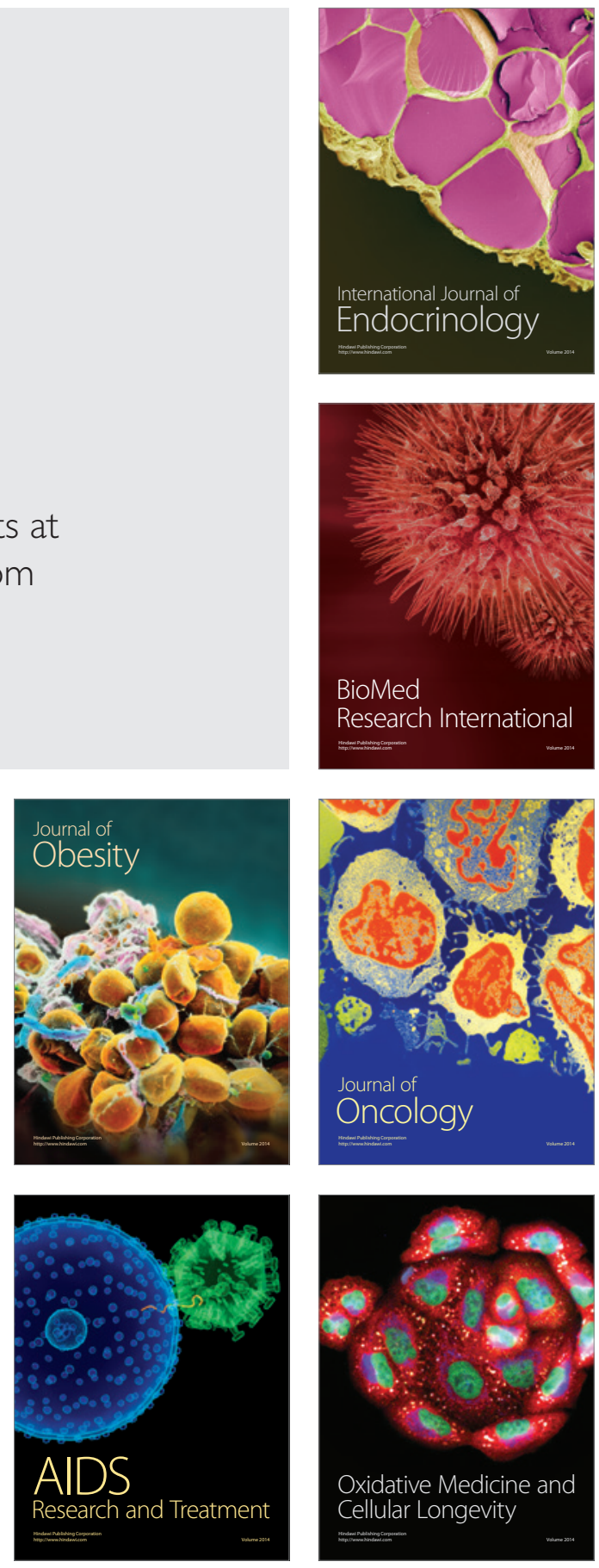